\title{
HaCIA UNA CARACTERIZACIÓN DE LOS TRADUCTORES E INTÉRPRETES en COLOMbia
}

\author{
Characterizing Translators and interpreters in Colombia
}

Pour une CARACTÉrisation des TRADUCTEURS ET INTERPRÈTES EN COLOMbie

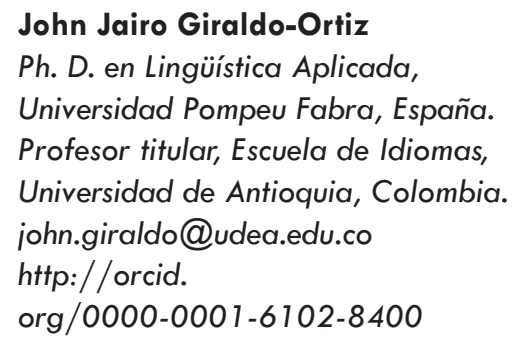

John Jairo Giraldo-Ortiz

Ph. D. en Lingüística Aplicada, Universidad Pompeu Fabra, España. Profesor titular, Escuela de Idiomas, Universidad de Antioquia, Colombia. john.giraldo@udea.edu.co http://orcid. org/0000-0001-6102-8400

Sandra Bibiana Clavijo-Olmos Ph. D. en Estudios de Traducción e Interpretación, Universidad de Alicante, España.

Profesora asociada, Universidad EAN, Colombia.

saclavijo@universidadean.edu.co https://orcid.

org/0000-0001-8493-8992

\section{Constanza Malavert-Chávez}

Magíster en Terminología, Universidad Pompeu Fabra, España. Traductora, ICONTEC, Colombia. constmal@yahoo.com https://orcid. org/0000-0003-0246-6069

\section{Bibiana Salazar-Giraldo}

Traductora Inglés-Francés-Español, Universidad de Antioquia, Colombia. Gestora de proyectos, Decoding TSL, Colombia.

bibiana.salazar@decoding.com.co https://orcid. org/0000-0002-2792-8795

\section{RESUMEN}

El objetivo principal de este estudio, de carácter exploratorio, metodología cuantitativa y enfoque descriptivo, fue realizar una caracterización delos traductores e intérpretes en Colombia. Se trata de un estudio que, junto con otros, servirá de base para que profesionales, agremiaciones y entidades gubernamentales tengan elementos para la definición de estrategias que les permitan a estos profesionales ser más competitivos en los contextos local y global. Para lo anterior, se contó con una muestra de 98 participantes de diferentes nacionalidades en el ejercicio de la profesión en Colombia, quienes respondieron una encuesta vía web. Entre los hallazgos se encuentran que la mayoría de quienes ejercen la profesión son mujeres, con edades entre 30 y 40 años, en modalidad de trabajo freelance, con residencia principalmente en Bogotá y Medellín, con formación superior en otras áreas de conocimiento, se dedican principalmente a la traducción científico-técnica, en su mayoría desconocen y por ende no aplican normas para el aseguramiento de la calidad de los servicios, y suelen desempeñar actividades complementarias como la docencia de traducción o lenguas extranjeras, la edición o la corrección de estilo. Estos hallazgos sugieren la necesidad de trabajar en la eliminación de por lo menos tres grandes riesgos que afectan localmente a la profesión, a saber: a) la escasa oferta de programas de profesionalización y formación continua; b) la baja cobertura del servicio, y c) la poca cultura de agremiación.

Palabras clave: traductores; interpretación; perfil del traductor; traducción en Colombia; caracterización del traductor.

\section{Abstract}

This exploratory study applied a quantitative methodology and a descriptive approach with the purpose to characterize Colombian translators and interpreters. This study, along with others, intends to provide professionals, associations and government entities the elements needed for creating strategies that allow them to be more competitive in local and global contexts. The sample consisted of 98 participants of different nationalities working in Colombia, who answered a survey via the web. The main findings show that most of practising translators are women, between 30 and 40 years of age, freelance, residing mainly in big cities as

Recibido: 2019-07-22/ Aceptado: 2020-06-08 / Publicado: 2020-09-17

https:/doi.org/10.17533/udea.ikala.v25n03a01 
Camilo Sarmiento-Jaramillo Magíster en Historia y teoría del arte, la arquitectura y la ciudad, Universidad Nacional de Colombia, Colombia.

Profesor asistente, Escuela de Ciencias Humanas, Universidad del Rosario, Colombia.

camilo.sarmiento@urosario.edu.co http://orcid.

org/0000-0002-7800-2853

El estudio reportado aquí fue llevado a cabo por el Comité Técnico 218 "Lenguaje y Terminología" del Instituto Colombiano de Normas Técnicas y Certificación (ICONTEC), el cual está conformado por varias instituciones de educación superior y empresas especializadas en estas áreas en Colombia.
Bogotá and Medellín, lack a culture of group action, such as in professional associations, have higher training in other areas of knowledge, are mainly engaged in scientific and technical translation, have low knowledge and use of standards for the assurance of service quality, and often work in complementary activities, such as translation or foreign languages teaching, editing and copyediting. These findings highlight the need to work on eliminating at least three major risks that affect the profession locally, as follows: (a) the limited offer of professionalization and continuing training programs; (b) the low coverage of the service, and (c) a lack of group action in professional associations.

Keywords: translators; interpretation; translator's profile; translation in Colombia; characterization of translators.

\section{RÉSUMÉ}

L'objectif principal de cette étude exploratoire, avec une méthodologie quantitative et une approche descriptive, a consisté à caractériser des traducteurs et interprètes colombiens. Il s'agit d'une étude qui, avec d'autres, servira de base aux professionnels, aux associations et aux entités gouvernementales pour disposer d'éléments nécessaires afin d'élaborer des stratégies permettant à ces professionnels d'être plus compétitifs tant au niveau local que mondial. Cette étude a été réalisée à partir rès d'un échantillon de 98 participants de différentes nationalités qui travaillent comme traducteurs et interprètes en Colombie. Ceuxci ont répondu à un questionnaire sur la web. Parmi les résultats, on constate que la majorité de ceux qui exercent la profession sont des femmes, âgées entre 30 et 40 ans, indépendantes, résidant principalement à Bogotá et à Medellín, avec un faible niveau de corporation, ayant suivi des études supérieures dans d'autres domaines de connaissance, se consacrant principalement à la traduction scientifique-technique, ayant une faible connaissance et utilisation des normes d'assurance de la qualité des services et qui travaillent généralement dans des activités complémentaires telles que l'enseignement de la traduction ou des langues étrangères, l'édition et la correction de style. Ces constats impliquent la nécessité de travailler sur l'élimination d'au moins trois risques majeurs qui affectent la profession localement, à savoir : a) l'offre limitée de programmes de professionnalisation et de formation continue ; b) la faible couverture du service, et c) le peu de culture du corporatisme.

Mots clés : traducteurs ; interprétation ; profil du traducteur ; traduction en Colombie; caractérisation des traducteurs. 


\section{Introducción}

Los traductores y los intérpretes han desempeñado funciones de vital importancia a lo largo de la historia: han inventado alfabetos, que han enriquecido la tradición escrita de diversas naciones; han promovido el desarrollo y la evolución de las lenguas, gracias a la introducción de neologismos y otras incorporaciones léxicas; han sido artesanos de la literatura y la han transformado con nuevas tradiciones y estilos; han sido difusores del conocimiento y han contribuido, así, al progreso de la ciencia; han sido actores en la escena del poder, aunque esto les haya valido condenas, censuras y discriminación; han propagado religiones, al llevar a los pueblos los textos sagrados en sus lenguas; han transmitido valores culturales, al importarlos y exportarlos de unas comunidades a otras; han sido creadores de diccionarios y han contribuido a la elaboración de enciclopedias; finalmente, han sido testigos privilegiados de la historia, incluso desde mucho tiempo antes de la invención de la escritura (Delisle y Woodsworth, 2005).

A pesar de la antigüedad de su profesión y la relevancia de esta para la evolución de las sociedades, los primeros registros de la traducción en la Nueva Granada datan del siglo XVIII (época en la que Colombia se llamaba de esa manera), con la traducción de los Derechos Humanos por parte de Antonio Nariño. Hoy en día existe aún poca bibliografía sobre el perfil del traductor en Colombia. Esta razón motivó al Comité Técnico 218 del ICONTEC a iniciar un estudio, en 2017, cuyo objetivo principal fue realizar una caracterización de los traductores e intérpretes en el país. Se trata de un estudio exploratorio, de metodología cuantitativa y enfoque descriptivo, que contó con una muestra de 98 participantes, quienes respondieron una encuesta autoadministrada vía web.

Este estudio, junto con otros, servirá de base para que tanto los profesionales y agremiaciones de estas áreas como el Gobierno puedan llegar a analizar los aspectos considerados como fortalezas y debilidades de la profesión, para definir las estrategias que les permitan a los profesionales de estas áreas ser más competitivos en los contextos local y global. Si se conocen las características de estos profesionales, se pueden buscar oportunidades para el gremio, que vayan desde la profesionalización, hasta la simple identificación de aspectos en los cuales se necesita mejor preparación. Reiteramos, pues, que es necesario contar con datos actualizados periódicamente que permitan describir el perfil del traductor y del intérprete en Colombia, para seguir trabajando, desde la academia y las asociaciones, en pro de la consolidación, el reconocimiento y la dignificación de la labor que desempeñan.

\section{Marco teórico}

Como primer paso, y con el fin de tener una línea de base acerca del estado general de la profesión en el contexto colombiano, se hizo un rastreo de estudios previos sobre el tema y se seleccionaron los más destacados. Estos trabajos iniciales abordan, cada uno desde su perspectiva, el perfil del traductor. El primero, publicado por Clavijo et al. (2008), de enfoque mixto y basado en una encuesta aplicada a 120 traductores colombianos en el año 2007, tuvo como finalidad recopilar información sobre el perfil del traductor, enfocado en aspectos como su lengua materna, lenguas de trabajo, dedicación a la labor traductora, rango de edad, formación, tiempo de experiencia, especialidades y recursos utilizados.

En 2018, este estudio fue complementado por Clavijo mediante la selección de una pequeña muestra de traductores. Entre sus hallazgos destaca que la combinación de lenguas más frecuente es inglés-español, con un $82 \%$, el $95 \%$ son traductores independientes, el rango de edad comprende a mayores de 50 años ( $44 \%)$, entre 40 y 49 años (30\%), entre 30 y 39 años (18\%) y entre 20 y 29 años (8 \%). En cuanto a las áreas sobre las que más se traduce, halló que, en su orden, son legal, comercial y de negocios. En definitiva, la autora encontró que el traductor actual es una persona que, en la mayoría de los casos, trabaja 
de forma independiente, con mayor demanda en el par inglés-español, que toma la certificación de traductor e intérprete oficial como garantía de calidad de sus servicios, ya que no requiere título profesional para desempeñar su oficio. Es una persona que no tiene un número fijo de horas diarias de dedicación a su trabajo; cuenta con experiencia en traducción y bagaje cultural, que ha adquirido viviendo en otros países; tiene un buen conocimiento de recursos tecnológicos, pero no posee un perfil académico definido.

El segundo estudio, llevado a cabo por Quiroz et al. (2013), concluye que ambas disciplinas son reconocidas como profesiones por el Estado, pero no reciben ningún tipo de garantías del mismo, ya que carecen de legislación para su protección. Además, los programas académicos ofrecidos son todavía muy escasos, al igual que los grupos de investigación y las agremiaciones o asociaciones. Este estudio encuentra una predominancia femenina que, según los autores, podría estar relacionada con la naturaleza liberal de la profesión. La modalidad más usual de trabajo es la independiente, y cuando laboran en el campo de la docencia, lo suelen hacer en la enseñanza de lenguas. Las principales lenguas de trabajo son el español, el inglés y el francés. Los autores identifican, en su estudio, dos asociaciones de profesionales en el área en Colombia: la Asociación Colombiana de Traductores, Terminólogos e Intérpretes (ACTTI) y el Colegio Colombiano de Traductores (ССT). Finalmente, una buena parte de las personas que ejercen estas actividades lo hacen de manera complementaria a otras ocupaciones, sin tener ningún tipo de formación en estas áreas, por lo que se crea una atmósfera de informalidad.

El tercer estudio, de Quiroz et al. (2015), se basa en dos investigaciones realizadas entre 2010-2013 y 2013-2014, respectivamente. Es de enfoque mixto e indaga sobre asuntos como formación profesional, información laboral y otros aspectos profesionales de los participantes. El resultado evidencia que la mayoría de los traductores se encuentra en las ciudades más densamente pobladas del país, como Medellín y Bogotá. En cuanto al rango de edad, muestra un mayor número de traductores activos entre los 36 y 40 años, con formación en docencia y lingüística, principalmente.

Los estudios antes señalados revelan realidades parciales del perfil de los traductores e intérpretes en Colombia. Cada uno observa, en momentos diferentes, ciertos aspectos relativos a estos profesionales en el país, pero sin llegar a una caracterización completa. Según nos consta, Colombia aún se encuentra en vías de definir el perfil pormenorizado de sus traductores e intérpretes. Por esta razón, el Comité Técnico $218 \mathrm{del}$ ICONTEC unió los esfuerzos de sus miembros, provenientes de la academia y del sector profesional, con el ánimo de contribuir al acopio y la actualización de información que conduzca a una caracterización más detallada de estos especialistas en el país. En síntesis, a partir de los hallazgos del estudio que se presenta aquí, se busca aportar información más actualizada que permita, a las partes interesadas, como las universidades, las asociaciones y el Comité Técnico 218, definir mejores acciones y estrategias para la proyección, el posicionamiento y el reconocimiento local del gremio.

\section{Método}

En este estudio se empleó la técnica de muestreo incidental o de conveniencia, que consiste en la selección directa e intencionada del público objetivo de la encuesta. Al aplicar este método, se buscaba aprovechar la ventaja de poder seleccionar a los encuestados, ya que los miembros del Comité Técnico 218 son, en su mayoría, docentes de traducción o personas relacionadas con traductores e intérpretes, lo cual facilitaba en gran medida su localización y le daba validez a la selección del público objetivo.

La Tabla 1 sintetiza las cuatro fases generales en que se desarrolló el trabajo. 
Tabla 1 Fases del estudio

\begin{tabular}{|c|c|c|c|}
\hline Fase & Tareas & Hito & Personas involucradas \\
\hline \multirow[t]{4}{*}{ 1. Diseño } & $\begin{array}{l}\text { Definición del problema y } \\
\text { enfoque }\end{array}$ & $\begin{array}{l}\text { Entrega del instrumento } \\
\text { (cuestionario de la encuesta) }\end{array}$ & $\begin{array}{c}\text { Miembros del Comité Técnico } 218 \\
\text { del Icontec }\end{array}$ \\
\hline & $\begin{array}{l}\text { Búsqueda de literatura } \\
\text { relacionada }\end{array}$ & & \\
\hline & Diseño del instrumento & & \\
\hline & $\begin{array}{l}\text { Validación y pilotaje del } \\
\text { instrumento }\end{array}$ & & \\
\hline 2. Recolección de datos & $\begin{array}{l}\text { Conformación de las listas } \\
\text { de distribución de correo } \\
\text { electrónico para envío de la } \\
\text { encuesta }\end{array}$ & Realización de la encuesta & \\
\hline \multirow[t]{2}{*}{ 3. Elaboración del informe } & Análisis de los datos & Entrega del informe & \\
\hline & Redacción del informe & & Experto en estadística \\
\hline 4. Difusión & $\begin{array}{l}\text { Redacción de artículo derivado } \\
\text { del informe }\end{array}$ & $\begin{array}{l}\text { Publicación en revista } \\
\text { especializada }\end{array}$ & $\begin{array}{l}\text { Miembros del Comité Técnico } \\
218 \text { del IcoNtec }\end{array}$ \\
\hline
\end{tabular}

En particular, la caracterización de los profesionales del área se llevó a cabo por medio de una encuesta, creada y validada por los traductores miembros del Comité Técnico 218, dirigida a traductores e intérpretes activos, de tiempo completo o parcial, que trabajan en calidad de independientes o asalariados en empresa, establecidos o con negocios en Colombia.

Con el fin de invitar a diligenciar el cuestionario, se envió un correo electrónico con la presentación, la justificación y el enlace respectivo para acceder a la encuesta, diseñada en Google forms. Este correo se remitió a los profesionales del área miembros de la ACTTI — que, para ese momento, contaba con 51 miembros-, y a 393 egresados de programas de Traducción de diferentes instituciones de educación superior, principalmente de la Universidad de Antioquia, Universidad Ean, Universidad del Rosario y Universidad Nacional.

Es de aclarar que, en primer lugar, prácticamente la mitad de las direcciones de correo de los egresados de la Universidad de Antioquia, alrededor de 150 , se encontraron inactivas. Esto se debe a que, para la época, esa institución tenía como política desactivar los correos institucionales de los estudiantes una vez egresaban de la carrera. Por consiguiente, esta situación produjo una reducción en el número de respuestas esperadas. En segundo lugar, en Colombia es complicado hacer un estudio que comprenda toda la población dedicada a la traducción e interpretación, dado que, según la "Clasificación industrial internacional uniforme de todas las actividades económicas", publicada por el Departamento Administrativo Nacional de Estadística (2012), los profesionales de estas áreas pueden registrarse bajo dos códigos de actividad económica diferentes: 6399 (Otras actividades de servicio de información) y 7490 (Otras actividades profesionales, científicas y técnicas). En consideración de todo lo anterior, el enlace para diligenciar la encuesta estuvo disponible durante el mes de noviembre de 2017 y se obtuvieron 98 respuestas. En consecuencia, se trata de una experiencia inicial de aproximación al conocimiento del perfil de los traductores; es una muestra de los resultados y análisis que se pueden hacer, para tener en cuenta en futuras mediciones. Por lo anterior, no se pretende que sea un perfil exhaustivo de la base de traductores e intérpretes, pero sí una contribución al establecimiento de este. 
Para la divulgación de la encuesta se buscaron mecanismos que aseguraran la difusión de la manera más amplia posible. En primer lugar, los miembros del Comité Técnico 218 enviaron la invitación a participar a los traductores e intérpretes que figuraban en sus listas de contactos, para no correr el riesgo de incurrir en violación de la Ley de Habeas Data o Ley Estatutaria 1581 de 2012, por la cual se dictan las disposiciones generales para la protección de datos personales en Colombia (Colombia, Congreso de la República, 2012). En segundo lugar, la información se difundió en el boletín virtual de la ACTTI, que fue publicado un mes antes del lanzamiento de la encuesta. En dicho boletín se anunciaba la encuesta y su propósito, de modo que los asociados se motivaran a participar en ella.

El cuestionario se elaboró a lo largo de varias reuniones del Comité Técnico 218 del icontec. Para ello, se tuvieron en cuenta aspectos como el perfil, la formación, el desempeño y la trayectoria profesional. Dicho cuestionario se sometió, antes de su distribución a la población objeto de estudio, a validación y pilotaje por parte de los siete miembros del Comité Técnico 218, que se dedican a labores de traducción o interpretación. Se leyó en voz alta, se ajustó la redacción de las preguntas y se hizo una prueba piloto entre algunos de los integrantes del comité.

El cuestionario final está formado por 3 partes, que contienen 41 preguntas, 18 de ellas abiertas y 23 cerradas. La primera parte indaga por datos sociodemográficos, como la edad, la nacionalidad y el sexo; la segunda, por la formación y los conocimientos de los traductores; y la tercera, por los aspectos relativos al ejercicio profesional y a la inserción en el mercado laboral. Para esto último, se incluyeron preguntas sobre las áreas de desempeño y especialización, la experiencia, las lenguas de trabajo, las formas de cobro y el conocimiento de normas que rigen el oficio.

Una vez que se cerró el periodo estipulado para diligenciar la encuesta, se procedió a descargar los datos de la plataforma, a desagregarlos para cada pregunta y a extraer las gráficas y tablas que permitían proceder al análisis de las respuestas. Este informe fue revisado por los miembros del comité y estuvo listo a finales del primer semestre de 2018.

Cabe destacar que el diligenciamiento del cuestionario se llevó a cabo de manera anónima. Esto protegió la identidad de los encuestados, la cual nunca fue solicitada ni revelada durante ninguno de los momentos del proceso.

Una vez que se tuvieron tabulados los datos, el comité contó con la presencia del experto en estadística, en dos de las reuniones del segundo semestre de 2018, con el fin de analizar la manera como se habían agrupado los datos y las respuestas en términos cuantitativos. El análisis cualitativo se efectuó en reuniones posteriores por los expertos en traducción e interpretación del comité, quienes ponderaron y discutieron los datos y establecieron las características básicas del perfil del traductor en Colombia, como puede apreciarse en el apartado siguiente.

\section{Resultados}

A continuación se presentan las características sociodemográficas que mostró la encuesta: sexo, edad, nacionalidad y lugar de residencia. De igual modo, se presentan datos acerca de la formación académica, áreas de desempeño, áreas de especialización, años de experiencia en el ejercicio de la profesión, vinculación a asociaciones y formas de cobro del servicio.

\section{Sexo}

De las 98 respuestas recibidas, el $68 \%$ (67) fueron enviadas por mujeres y el $32 \%$ (31) por hombres, lo cual muestra claramente la predominancia del sexo femenino entre quienes ejercen la profesión de traductor e intérprete en Colombia, dato que se constata igualmente en otros estudios, como los de Clavijo y Duro (2020), Ministerio de Cultura de España y TT ACE Traductores (2010), Quiroz et al. (2015) y Valdez (2018). 


\section{Edad}

Para analizar la edad de quienes respondieron la encuesta, se clasificaron las respuestas en rangos de 10 años, desde los 20 hasta los 60 años, y se añadió una categoría para incluir a los mayores de 60 años. Si se estudian desde este punto de vista las respuestas, se encuentra que $26 \%$ de los encuestados tiene de 20 a 30 años; $31 \%$, de 30 a 40 años; $20 \%$, de 40 a 50 años; $8 \%$, de 50 a 60 años, y $15 \%$ es mayor de 60 años. Estos datos permiten inferir que el perfil ocupacional de los oficios de traductor e intérprete muestra que estos tienden a ser ejercidos por personas menores de 40 años, pues el $57 \%$ de los encuestados pertenecen a estos rangos etarios. Por el contrario, solo el $23 \%$ de la muestra corresponde a personas mayores de 50 años.

\section{Lugar de residencia}

Si se hace énfasis en la oferta de servicios de traducción e interpretación de acuerdo con los lugares de residencia de quienes la llevan a cabo, se obtiene que la mayoría de los encuestados habitan en las dos ciudades principales del país, Bogotá (51\%) y Medellín y el área metropolitana (32\%), esto es, el $83 \%$ del total de la muestra. En el resto de Colombia apenas habitan 9 personas dedicadas al oficio, lo cual supone un $9 \%$ de los encuestados, y otras $8(8 \%)$ viven en el exterior. Estos datos llevan a pensar que el mercado está concentrado en las dos ciudades más pobladas del país, lo que supone que otros grandes centros urbanos y económicos como Cali, Barranquilla, Cartagena, Bucaramanga y Pereira bien podrían desarrollar una oferta competitiva en el área.

Si se cruzan los resultados obtenidos en las categorías correspondientes a lugar de residencia y sexo, se refuerza la idea del predominio femenino en el oficio. Tanto en Bogotá como en Medellín, al igual que en el resto de Colombia y en el exterior, el número de mujeres es superior al de hombres. En Bogotá, respondieron 38 mujeres (76\%) y 12 hombres (24\%); en el resto de Colombia, 7 mujeres (78 \%) y 2 hombres (22\%); y en el exterior, 5 mujeres (63\%) y 3 hombres (37\%). Esta distancia entre los dos sexos, notable en el resto de lugares de residencia, se acorta en Medellín, donde respondieron 17 mujeres ( $55 \%$ ) y 14 hombres ( $45 \%$ ), lo que permite colegir que hay tendencia a la igualdad de participación de ambos sexos en el oficio en esta última ciudad.

Si se cruzan los datos sobre el lugar de residencia con la edad, se observa que el oficio tiende a ser ejercido por menores de 40 años en la mayoría de lugares, con excepción de Bogotá, donde el $52 \%$ de los encuestados manifestó ser mayor de 40 años, contra el $48 \%$ que es menor de esa edad. En la capital, de hecho, residen 11 (78,5\%) de las 14 personas mayores de 60 años que respondieron la encuesta, lo cual muestra que, en esta ciudad, se encuentran los traductores e intérpretes de mayor edad.

\section{Nacionalidad}

Cuando fueron indagados acerca de su origen, 89 de los encuestados respondieron ser colombianos; es decir, el $91 \%$ de la muestra. El $15 \%$ de los encuestados ( 15 personas) afirmó tener una nacionalidad extranjera. Esto se explica porque 6 personas dijeron tener otra nacionalidad aparte de la colombiana y 9 tienen solamente nacionalidad extranjera.

Entre los países de procedencia de quienes afirman tener una nacionalidad distinta a la colombiana se destaca Estados Unidos, que cuenta con cinco nacionales. Los demás países representados son Venezuela, con dos personas, y Argentina, Brasil, Canadá, España, Francia, Italia, México, y Suiza, cada uno con un nacional.

\section{Formación}

En esta encuesta también se indagó por el grado de formación de los traductores e intérpretes. Del total de los encuestados, 38 \% declaró tener estudios de pregrado en traducción e interpretación; $19 \%$, especialización; $8 \%$, maestría, y $2 \%$, diplomado. En contraste, $32 \%$ de los encuestados 


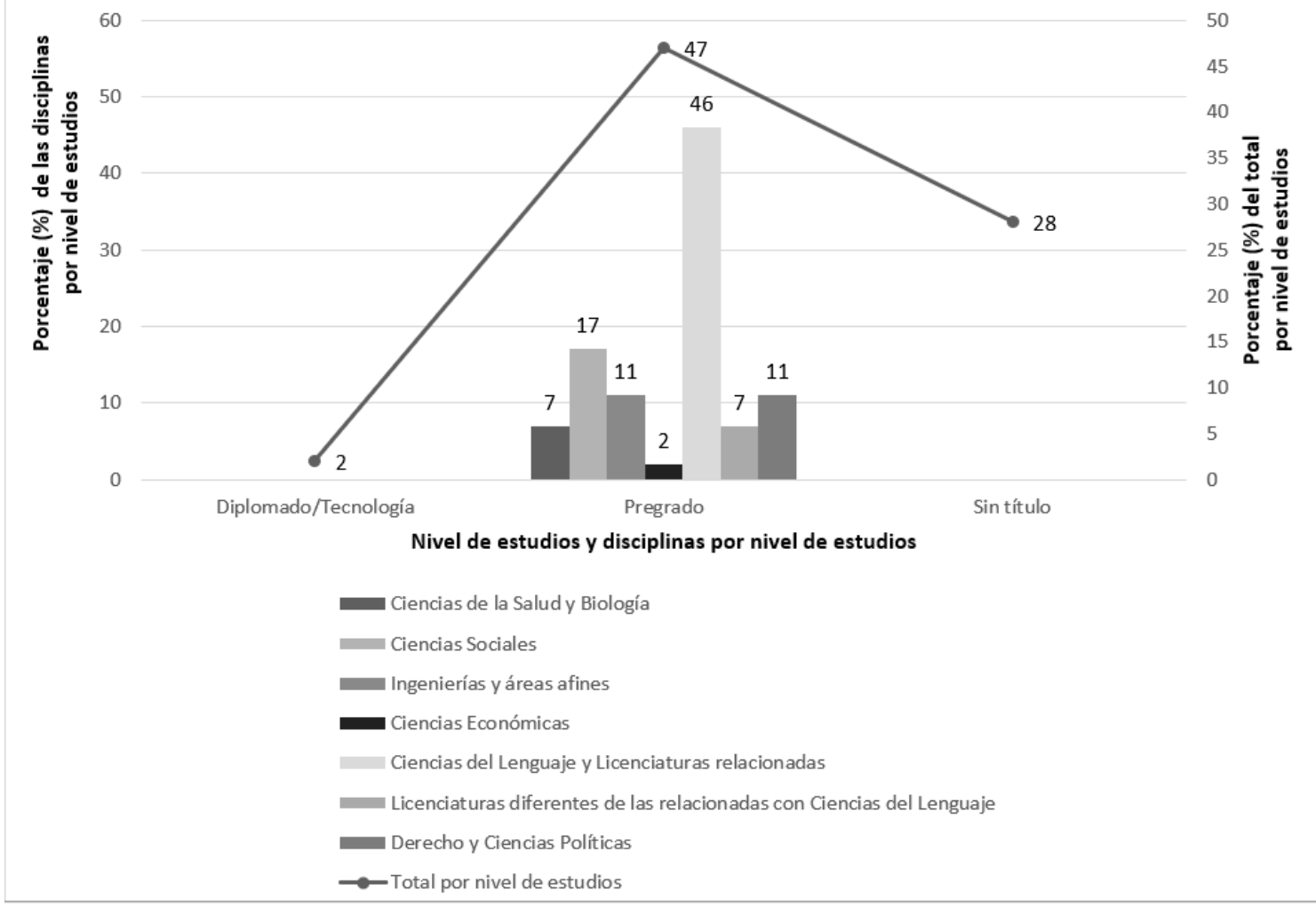

Figura 1 Nivel de estudios de los encuestados $v$ s. áreas de conocimiento diferentes a traducción (pregrado)

manifestó no tener ningún título y el $1 \%$ no especificó su nivel de formación.

Adicionalmente, respecto a títulos de áreas diferentes a la traducción e interpretación, $47 \%$ de los traductores encuestados expresó poseer título de pregrado, principalmente en las áreas de Ciencias del Lenguaje y licenciaturas relacionadas, Ciencias Sociales, Ingenierías y áreas afines y Derecho y Ciencias Políticas. El 2 \% indicó haber cursado un diplomado o tecnología (sobre traducción en ámbitos de negocios y administración de empresas, respectivamente). En contraste, el $28 \%$ manifestó no tener ningún título académico (véase Figura 1). En cuanto a formación posgraduada, $18 \%$ dijo tener título de maestría; el $1 \%$, de doctorado, y el $4 \%$, de especialización. Las áreas más comunes en la formación posgraduada son Ciencias del Lenguaje, Ciencias Económicas, Ciencias Humanas, Derecho y Ciencias Políticas y Ciencias de la Salud y Biología. (véase Figura 2).
En lo concerniente a cursos complementarios, $56 \%$ de los traductores encuestados afirmó haber hecho cursos de corrección, estilo y redacción. En cuanto a la frecuencia con que realiza cursos de actualización, $34 \%$ manifestó que lo hace una vez al año; $31 \%$, dos o más veces al año; $12 \%$, cada dos años, $4 \%$, cada cinco años. En contraste, $19 \%$ respondió que nunca ha participado en cursos de actualización. Lo anterior evidencia que una tercera parte de la población objeto de estudio procura capacitarse, tomando al menos un curso al año.

\section{Áreas de desempeño}

El $47 \%$ de los encuestados manifiesta tener como actividad principal la traducción, en tanto que $11 \%$ se dedica mayoritariamente a la interpretación. En cuanto a la especialidad en la que se suele desempeñar, $23 \%$ se dedica a la traducción oficial hacia el español, lo cual tiende a coincidir con el estudio de Zuluaga y Quiroz (2018, p. 281), que muestra que $4(28,57 \%)$ de los 14 traductores e 


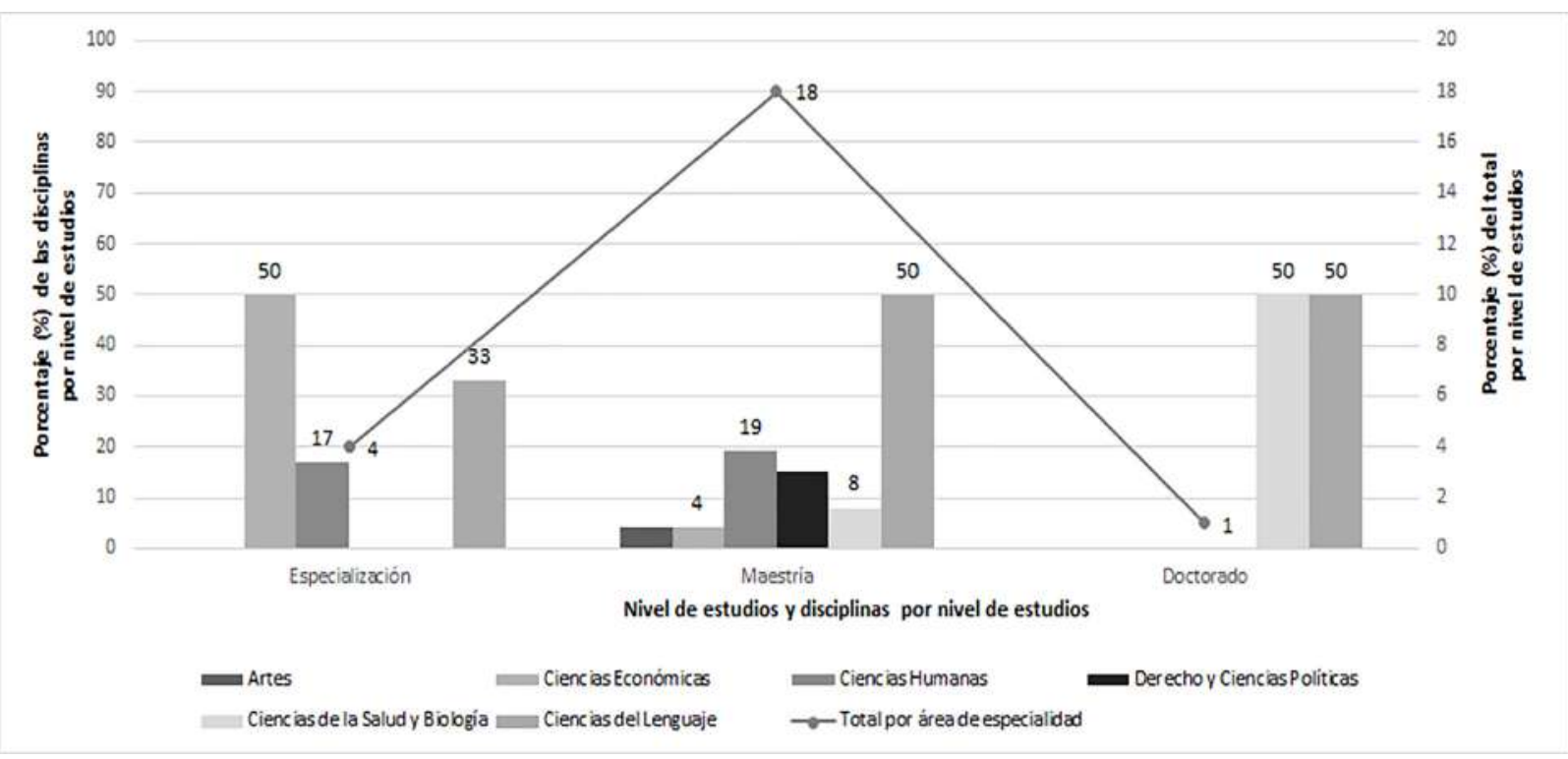

Figura 2 Nivel de estudios de los encuestados vs. áreas de conocimiento diferentes a traducción (posgrado)

intérpretes oficiales certificados hasta el momento por la Universidad de Antioquia lo hacen del inglés al español.

Nuestro estudio muestra, además, que $25 \%$ de los encuestados se dedica a la traducción oficial hacia una lengua diferente al español; $10 \%$, a la traducción literaria; $3 \%$, a la traducción literaria hacia una lengua distinta del español; $36 \%$, a la traducción especializada hacia una lengua distinta del español; $58 \%$, a la edición y la corrección de textos en español, y en lo que respecta a docencia, $68 \%$ de los encuestados sostiene que se ha desempeñado como docente de lenguas, y $31 \%$, como docente de traducción.

\section{Experiencia}

En lo referente al tiempo de ejercicio de la profesión, en promedio, la experiencia de quienes contestaron la encuesta se ubica en 10 años y 4 meses.

En cuanto a la modalidad de trabajo, $91 \%$ indicó que trabaja como traductor independiente, $8 \%$ como traductor de planta en una empresa y $1 \%$ como traductor interno, es decir, en una agencia de traducción.

Así mismo, $91 \%$ manifestó que trabaja para clientes locales y $9 \%$ para clientes globales exclusivamente.
El 58\% indicó que trabaja tanto para para clientes locales como globales.

El $32 \%$ de los encuestados afirmó trabajar en los campos en los que se ha especializado, entre los que sobresalen áreas como: medicina, derecho, educación, ingeniería, economía y finanzas, tecnología, entre otras. El $68 \%$ restante sostuvo que trabaja en la traducción de textos según la demanda que presente el mercado. Infortunadamente, sobre este último aspecto no se indagó en la encuesta; por consiguiente, no se logra aportar datos al respecto.

No obstante, de acuerdo con datos reportados en 2019 por la Agencia de Traducción e Interpretación de la Universidad de Antioquia (vía correo electrónico), se tiene que, en el contexto de la ciudad de Medellín, los servicios de traducción más demandados son, en su orden, la traducción oficial $(82,39 \%)$ y la traducción técnica $(12,71 \%)$. El 4,9 \% restante corresponde a servicios diferentes a la traducción.

\section{Filiación}

La filiación de los traductores e intérpretes a alguna asociación es una parte importante en su desarrollo profesional, pues, en general, este tipo de asociaciones ofrece a sus miembros cursos de 
actualización en muchas áreas de interés profesional, al existir una oferta mínima de educación formal en estos campos por parte de las universidades en Colombia. A la pregunta sobre la filiación, el $69 \%$ de los encuestados respondió que no pertenecen a ninguna asociación; $24 \%$ pertenecen a la ACTTI; $4 \%$, a la American Association of Translators (ATA); 3 \%, al Colegio Colombiano de Traductores (ССТ). Así mismo, del total de encuestados, el $6 \%$ manifestó pertenecer además a alguna de las siguientes asociaciones: Asociación Española de Traductores, Correctores e Intérpretes (ASETRAD), Sección Autónoma de Traductores de Libros de la Asociación Colegial de Escritores de España (ACE traductores), Association of Translators and Interpreters of Florida (ATIF) o National Association of Judiciary Interpreters and Translators (NAJIT).

\section{Áreas de especialización}

En la encuesta se preguntaba también acerca de las áreas de especialización de los profesionales. Es importante tener en cuenta que, en muchos casos, los encuestados identificaron más de un área de especialización, razón por la cual los porcentajes no son acumulativos para el conjunto de áreas de especialización.

Con el fin de presentar valores representativos de cada ítem analizado, se tomaron los porcentajes superiores al $20 \%$ y, en algunos casos, porcentajes menores cuando hacía referencia a algún ítem específico que se deseaba analizar.

Las áreas de especialización con una proporción más alta fueron la técnica (27\%) y la científica (22\%), respectivamente. Llama la atención que, en el tercer lugar, con un porcentaje del $23 \%$, se encuentran aquellas personas que informan no tener área de especialización. En su orden, las siguientes áreas que mencionaron los encuestados son: ciencias sociales y humanas $(14 \%)$, jurídica (13\%) y económica (9\%). El $20 \%$ no contestó a esta pregunta.

Con respecto a la traducción literaria, $10 \%$ de los encuestados traduce o interpreta hacia el español, y $3 \%$, hacia una lengua distinta del español. El $8 \%$ de los encuestados firma contratos con editoriales para recibir derechos sobre la traducción.

En cuanto a la traducción oficial, el $23 \%$ de los encuestados trabaja hacia el español, y el $25 \%$, hacia una lengua distinta del español.

Desde el punto de vista de los grupos etarios, en el rango de 20 a 30 años, el $32 \%$ de los encuestados se dedica a la traducción técnica, con un porcentaje igual para quienes no tienen área de especialización, seguidos por quienes manifiestan trabajar en traducción científica (20\%).

En el rango de 31 a 40 años, $17 \%$ indicó que trabaja en traducción científico-técnica, $27 \%$ contestó no tener un área de especialización definida y $43 \%$ no respondió a esta pregunta.

En el rango de 41 a 50 años, los porcentajes se encuentran distribuidos de manera bastante uniforme entre la traducción técnica (35\%), científica (35\%), ciencias sociales $(25 \%)$ y, por último, económica $(20 \%)$.

En el rango de 51 a 60 años, $25 \%$ trabaja en las áreas científico-técnica y literaria, respectivamente. Idéntico porcentaje representan quienes respondieron no tener un área de especialización. Cierran este grupo, aunque en menor grado, quienes afirmaron dedicarse a la traducción oficial, con $13 \%$.

Finalmente, en el grupo de traductores de más de 60 años, el área de especialización principal es la de traducción técnica (47\%), seguida por la traducción jurídica (33\%), “otra” (27\%) y la traducción económica (20\%).

\section{Area de especialización y zona de residencia}

Sobre la zona de residencia, $30 \%$ de los encuestados residentes en Bogotá respondió que se dedica a la traducción técnica y $22 \%$ a la traducción científica. El $20 \%$ mencionó no tener área de especialización. El 28\% restante se dedica a otros tipos de traducción, entre los que se encuentran la jurídica, oficial, económica y audiovisual. 
El 29\% de los encuestados residentes en Medellín y su área metropolitana respondió que no tiene área de especialización; el $26 \%$ se dedica a la traducción científico-técnica, y $19 \%$ presta sus servicios en alguno de los siguientes tipos de traducción: jurídica, económica, oficial, técnica. El $26 \%$ restante no contestó a esta pregunta.

El $44 \%$ de los encuestados que residen en el resto de Colombia no respondieron, mientras que $22 \%$ afirmaron que trabajan en traducción de textos científico-técnicos y de ciencias sociales y humanas. El 34\% restante indicó que trabaja en traucción de algunos de los siguientes tipos de textos: económicos, jurídicos, oficiales, entre otros.

Por último, $38 \%$ de los encuestados que residen en el exterior respondieron que no tienen área de especialización; $13 \%$ dijeron ser especialistas en traducción científico-técnica; $24 \%$ manifestó que se desempeña en alguna de las siguientes áreas: económica, jurídica, literaria, entre otras, y $25 \%$ no respondió esta pregunta.

\section{Área de especialización y sexo}

En relación con el área de especialización según el sexo al que pertenecen los participantes, el tipo de traducción que más se destaca es la científico-técnica, con $44 \%$ de las mujeres y $56 \%$ de los hombres que contestaron la encuesta, respectivamente. Por otro lado, $24 \%$ de mujeres y $23 \%$ de hombres indicaron que "no tienen área de especialización".

A continuación se presentan los últimos epígrafes de este apartado, que abordan las principales modalidades de interpretación, edición y corrección de textos, formas de cobro, trabajo con revisores o correctores, y conocimiento y aplicación de normas técnicas en el campo de la traducción.

\section{Interpretación como área de especialización (modalidades y lenguas de trabajo)}

La encuesta incluía una pregunta dirigida a conocer la dedicación de los intérpretes a su actividad.
En este sentido, se encontró que, del total de encuestados, solo $11 \%$ se dedica a la interpretación como principal actividad profesional. Este hecho nos hace plantearnos las dos hipótesis siguientes:

1. El mercado de la interpretación requiere solamente un $11 \%$ de intérpretes a tiempo completo y un $89 \%$ en forma parcial.

2. El $89 \%$ ejerce la interpretación como una segunda actividad, porque no tiene las competencias profesionales suficientes para realizar actividades de interpretación.

En el primero de los casos, no es posible abordar esta situación, ya que es el mercado el que determina la oferta de servicios de interpretación. Sin embargo, en el segundo caso es posible proveer la formación profesional necesaria en interpretación, de acuerdo con la demanda del mercado y las competencias y exigencias de la profesión.

Por tipos de interpretación, la encuesta mostró los siguientes datos: en interpretación consecutiva, sobresale el trabajo en los pares de lenguas inglésespañol (20\%), seguido de español-inglés (14\%), portugués-español (5\%), francés-español (4\%), español-francés (3\%) y otros pares de lenguas, entre los que se encuentran el alemán, el italiano y el chino (6\%). El $48 \%$ restante indicó no trabajar en esta modalidad de interpretación.

En interpretación simultánea, los pares de lenguas con mayor porcentaje siguen siendo inglés-español (19\%) y español-inglés (14\%), seguidos de lejos por los pares portugués-español (4\%), español-francés (3\%) y francés-español (2\%) (véase Figura 3a). Al igual que en la consecutiva, los pares de idiomas que involucran el alemán, el italiano y el chino son los menos ofrecidos (1\%).

En interpretación de acompañamiento, los pares de lenguas con mayor porcentaje son nuevamente inglés-español (23\%) y español-inglés (14\%), seguidos por el francés ( $4 \%$ ). Los pares de idiomas 
$a$

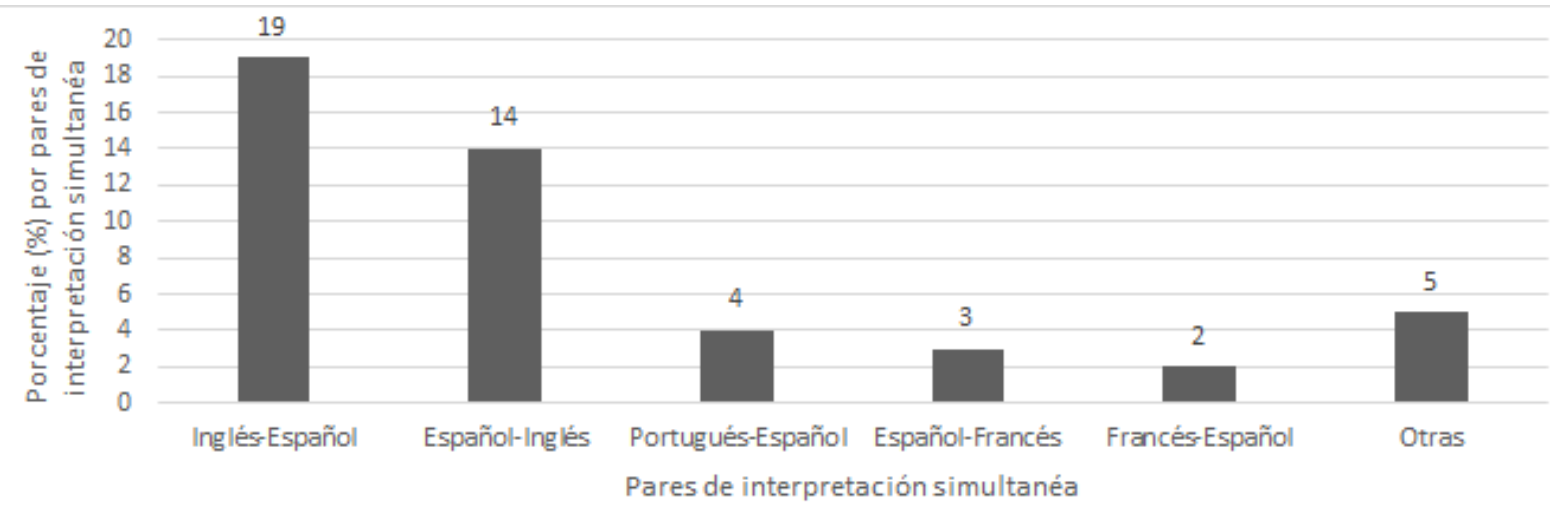

$b$



Figura 3 Pares de lenguas con mayor oferta en interpretación simultánea $(a)$ e interpretación de acompañamiento $(b)$

que involucran el italiano y el alemán solo tuvieron el $1 \%$ (véase Figura $3 b$ ).

Los resultados relacionados con interpretación jurada o en corte mostraron una oferta mayor en los pares de lenguas inglés-español $(8 \%)$ y español-inglés $(6 \%)$, seguidos por francés-español y español-francés $(2 \%)$ e italiano (1\%), en ambas direcciones. Se encontró, igualmente, que en la actualidad no hay oferta de este servicio para el idioma chino.

Por último, en lo que respecta a la interpretación comunitaria, los pares de lenguas más ofrecidos son español-inglés (5\%), inglés-español (4\%), francés-español (3\%) y portugués-español (2\%).

\section{Edición y corrección de textos como área de especialización}

En la encuesta se indagó, además, por el tema de edición y corrección de textos, conceptos conocidos por los encuestados y definidos en la norma técnica colombiana (NTC)-ISO 17100 (ICONTEC, 2017a).

Del total de encuestados, $58 \%$ ofrece estos servicios en español, $27 \%$ en inglés y $5 \%$ en francés. Los encuestados informaron que los trabajos de este tipo solicitados con mayor frecuencia son los relacionados principalmente con textos académi$\cos (6 \%)$ y técnicos $(5 \%)$. 


\section{Trabajo con pares revisores o correctores}

Respecto de la pregunta acerca de si los traductores trabajan con algún colega traductor o corrector, $59 \%$ respondió afirmativamente. En este caso, se hace referencia a traducción compartida y, a su vez, a corrección y revisión de traducciones. Este hecho constituye una buena práctica dentro de la profesión y demuestra el esmero por mantener altos estándares de calidad en el trabajo.

Con el lanzamiento de la norma técnica NTCIso 17100 en Colombia, se espera que los traductores trabajen con mayor frecuencia de manera colaborativa, cumpliendo así con los estándares de la norma en cuanto a revisión y edición de traducciones.

\section{Formas de cobro}

En este ítem se halló que las respuestas son más homogéneas, pues en las variables que se examinaron (lugar de residencia y edad), la opción empleada con más frecuencia es el cobro por palabra, que alcanzó el $85 \%$ en el rango de 51 a 60 años, y $80 \%$ en el de más de 60 años. Por zonas de residencia, este método de cobro alcanzó $74 \%$ en Medellín y el área metropolitana, y $72 \%$ en Bogotá.

En síntesis, se encontró que $68 \%$ de los traductores encuestados cobra por palabra, $13 \%$ por página, $3 \%$ por cuartilla y el $2 \%$ por carácter. El $14 \%$ no respondió a esta pregunta. Estos datos muestran que la forma de cobro de traducciones, por palabra, es ya una manera más uniforme en el ámbito nacional e internacional.

En relación con la tarifa de los intérpretes, $11 \%$ cobra por día y $21 \%$ cobra por hora. El $70 \%$ de los intérpretes no contestó esta pregunta, tal vez por la manera en que se conformó este gremio en Colombia, a partir de la experiencia más que de la formación. Es un grupo de profesionales competitivo, que suele ser muy reservado a la hora de divulgar sus tarifas, quizás por el temor a la competencia desleal y la consecuente pérdida de clientes que buscan un servicio de calidad, pero más barato; por la gran experiencia que tiene, o debido a la falta de agremiación, que sí permite unificar criterios al respecto.

\section{Conocimiento y uso de normas}

Las últimas preguntas de la encuesta hacen referencia al conocimiento de normas técnicas nacionales e internacionales por parte de los encuestados. El $26 \%$ manifestó conocerlas y el $86 \%$ reconoció que aplicar dichas normas le daría valor a su portafolio de servicios.

Finalmente, en lo que respecta a las normas que conocen y aplican, $11 \%$ de los traductores respondió que usa la NTC 5808 (actualmente NTC-ISO 17100) sobre "Servicios de traducción. Requisitos para la prestación del servicio" (ICONTEC, 2017a), $2 \%$ usa la norma Iso 704 sobre "principios y métodos del trabajo terminológico” (ICONTEC, 2013), 4 \% emplea otras normas y $10 \%$ no usa ninguna norma.

\section{Discusión}

Los estudios sobre el perfil y el estatus del traductor y el intérprete en Colombia apenas están dando sus primeros pasos, lo que es comprensible si se tiene en cuenta que el país solo cuenta con: 1) cuatro programas académicos de formación en traducción (ninguno en interpretación): un pregrado en la Universidad de Antioquia, una especialización en la Universidad del Rosario, y dos maestrías, una en la Universidad de Antioquia y otra en la Universidad Autónoma de Manizales; 2) cuatro grupos de investigación relacionados con traducción, pertenecientes a las universidades de Antioquia, Autónoma de Manizales, del Valle e Industrial de Santander (Colombia, Ministerio de Ciencia, Tecnología e Innovación, 2020); y 3 ) un par de asociaciones, la ACTTI y el сCT. No obstante, los escasos estudios existentes sobre el perfil y el estatus de los traductores permiten esbozar cierta similitud en el comportamiento de estas profesiones en diferentes momentos y contextos, sobre todo en lo que respecta a variables sociodemográficas y a características relacionadas con la 
formación, vinculación, dedicación y agremiación de quienes las desempeñan.

Los resultados del presente estudio muestran datos que aportan a la caracterización de quienes laboran actualmente en los campos de la traducción e interpretación en Colombia. A grandes rasgos, se encuentra una tendencia al predominio del sexo femenino en el ejercicio de la profesión (68\%). De acuerdo con Gouadec (2010), las razones de esto pueden ser económicas y sociales. Dado que la profesión es flexible, en términos de horarios y de lugar de trabajo, los ingresos se consideran un complemento a la economía familiar. Esta tendencia se observa en otros países, como, por ejemplo, México (61,7 \%) y España (54,1 \%) (Fundación Italia Morayta, 2017; Lozano, 2011). Al respecto, Clavijo y Duro (2020, p. 358) citan informes como el de la Asociación Europea de la Industria de la Lengua (European Language Industry Association, 2019), que habla de un $73 \%$ de mujeres y, de manera anecdótica, incluye también la información de México, donde el informe de la Fundación Italia Morayta, de 2017, identifica un 61,7 \% de mujeres y habla de la única excepción en relación con las lenguas indígenas, donde hay un 5,79\% más hombres que mujeres.

La mayoría de las personas que se dedican en Colombia a las profesiones bajo estudio se ubican en la franja etaria de 30-40 años, con un promedio de 39,11 años. Adicionalmente, se ha encontrado que estas profesiones son ejercidas en su mayoría por personas de nacionalidad colombiana y con una experiencia promedio de 10,4 años. Se ha evidenciado alta concentración de la oferta del servicio en las dos principales ciudades, es decir, Bogotá y Medellín. Esto se explica principalmente por el tamaño de su población y por su condición de grandes centros económicos, financieros, industriales y políticos, que demandan mayor variedad de servicios de traducción e interpretación y de pares de lenguas.

La modalidad de trabajo predominante es la freelance y sus clientes son, por lo general, nacionales.
Suelen cobrar por palabra (traductores) o por horas (intérpretes), y tan solo el $31 \%$ manifestó estar agremiado.

La mayoría de quienes tienen estudios en traducción e interpretación han obtenido títulos de pregrado y especialización. Por su parte, aquellos que ejercen la profesión, pero que provienen de otras áreas, como lenguas modernas, derecho, ciencias políticas, ingeniería, educación, comunicación, literatura y filosofía, por citar las más comunes, han manifestado tener principalmente títulos en pregrado y maestría. Así mismo, más de la mitad de los encuestados indicó que se capacita, por lo menos una vez al año, mediante cursos complementarios, en especial de redacción, edición y corrección de estilo.

Además, llama la atención el alto desconocimiento y el uso de las normas técnicas de traducción, pues se encontró que menos del $15 \%$ de los encuestados dice conocerlas y aplicarlas, en especial la norma técnica colombiana NTC-ISO 17100, referente a los servicios de traducción y los requisitos para la prestación del servicio.

La mayoría de los encuestados dice laborar en el campo de la traducción como su actividad principal. En cuanto a las especialidades en las que se suelen desempeñar, sobresalen traducción especializada hacia una lengua distinta del español, edición y corrección de textos en español, traducción oficial hacia el español, traducción oficial hacia una lengua diferente al español y traducción literaria hacia el español. El $77 \%$ de los traductores e intérpretes también suele dedicarse a la docencia tanto de lenguas extranjeras como de traducción.

La traducción científico-técnica ocupa el lugar preponderante, siendo las áreas de especialización más comunes las ciencias sociales, el derecho y la economía. Sin embargo, cabe destacar que la mayoría de los traductores indicó que trabajan con textos de cualquier campo de especialidad que les imponga el mercado; por tanto, su perfil los hace versátiles. 
Ante la falta de oferta académica suficiente en el país y de aplicación de las normas técnicas, que brindan las especificaciones básicas sobre los servicios de traducción (NTC-ISO 17100 y GTC 283) y de los servicios de interpretación (NTC-ISO 18841) (ICONTEC, 2017a, 2017b, 2018), es necesario, como bien lo afirma Morón (2010, p. 15), reforzar, desde los centros de formación, la preparación en competencias, pues estas permiten al estudiante convertir en específicas aquellas competencias que antes fueron transversales. Esto sitúa a las competencias transversales en el núcleo de la formación a lo largo de la vida, en su capacidad de reciclaje y adaptación a las condiciones del medio en el que se desenvuelven.

En cuanto a los tipos de interpretación, se destaca la oferta de interpretación consecutiva, principalmente en el par inglés-español. En lo que respecta a los tipos de interpretación simultánea, de acompañamiento y jurada, al igual que en el caso anterior, el par de lenguas más ofertado es inglés-español, mientras que, en el caso de la interpretación comunitaria, predomina la oferta en la vía español-inglés.

Otro punto que merece destacarse, como se ha ido esbozando en apartados precedentes, es el poco conocimiento que hay, entre quienes se dedican en Colombia a las actividades de mediación lingüística, de las normas técnicas nacionales e internacionales sobre traducción, interpretación y terminología. Lo anterior implica que no se aprovechen de forma óptima los beneficios que trae la normalización para los diversos sectores productivos. Esta falta de conocimiento y de aplicación de las normas se debe fundamentalmente a dos razones: 1) el desconocimiento de ellas por parte de los traductores, y 2) la creencia errónea de que las normas técnicas se equiparan con los reglamentos técnicos de obligatorio cumplimiento. ${ }^{1}$

1 Se suele confundir ambos tipos de documentos, cuando, en realidad, las normas técnicas son documentos elaborados por un organismo de normalización y son de aplicación voluntaria, mientras que los reglamentos
El Comité Técnico 218 del ICONTEC ha realizado labores de difusión nacional de los documentos sobre traducción, pero es consciente de la necesidad de lograr una mayor sensibilización, entre los traductores e intérpretes, acerca del papel fundamental de normas técnicas como la NTCISO 17100 y la NTC-ISO 18841 para mejorar ciertos aspectos de la calidad, que permitan, a su vez, mejorar el posicionamiento, prestigio y reconocimiento de la profesión.

Por último, los encuestados que manifestaron ofrecer el servicio de edición y corrección de textos lo hacen principalmente para textos académicos y técnicos escritos en español y, en menor medida, en inglés y francés.

\section{Conclusiones}

La importancia de estudios como este radica en que, gracias a su aporte en el conocimiento del perfil de los profesionales de la traducción e interpretación, se pueden buscar oportunidades de mejora para la profesión, que van desde la profesionalización hasta la identificación de los aspectos en los que se requiere actualización o mayor capacitación. Estudios de este corte sirven, sin duda, como insumo en la toma de decisiones para el mejoramiento del currículo en los programas académicos, presentes y futuros, que se ofrezcan en el país.

El hecho de que varios traductores e intérpretes manifestaran que su formación se dio en áreas diferentes, principalmente en lenguas modernas, derecho, ciencias políticas, ingeniería, educación, comunicación, literatura y filosofía, confirma el fenómeno señalado por Aguayo en el sentido de que la traducción y la interpretación tienen carácter interdisciplinario, lo que desdibuja los límites de la profesión. En concreto, sugiere lo siguiente:

técnicos son elaborados por el Gobierno y son de aplicación obligatoria. 
La asunción de la TI [Traducción e Interpretación] como campo interdisciplinar en el propio mercado laboral está suscitando toda una serie de cuestionamientos de la propia identidad del traductor y de la traducción e interpretación hoy. Así, detectamos una sensación generalizada de que la profesión del traductor se caracteriza por tener contornos borrosos (Kuznik 2011; Dam y Koskinen 2016a: 2), en continua redefinición (Dam y Koskinen 2016b: 257), siguiendo la concepción de la actividad de traducción de Mayoral (2004: 166) como una tarea compleja y polifacética (Aguayo, 2017, p. 20).

Coincidimos con Hortal (2010), quien afirma que "la sociedad marca lo que necesita y espera de cada profesión, configura su imagen social, ofrece alicientes, determina en qué lugares se ejerce la profesión, con qué medios, bajo qué leyes y normas" (p. 230). En este sentido, si bien es cierto que la traducción y la interpretación tienen el reconocimiento del Estado colombiano (aunque mediante una legislación insuficiente), ${ }^{2}$ este estudio ha evidenciado básicamente cuatro grandes riesgos que la profesión debe atender y eliminar para garantizar su dignificación y, por consiguiente, el alcance de un estatus adecuado en la sociedad:

1. Escasa oferta de programas para la profesionalización y la capacitación en aspectos que van desde el emprendimiento, las normas para el aseguramiento de la calidad en los procesos de traducción, interpretación y terminología, hasta el manejo de software especializado.

El aspecto de la capacitación es más sensible en la interpretación, pues la oferta académica es inexistente en el país. Esta situación podría ocasionar ineficiencia en el trabajo, además de afectar potencialmente la precisión y, por consiguiente, la calidad del trabajo de mediación comunicativa efectuado por los traductores e intérpretes.

2 Colombia no cuenta aún con una ley que reconozca verdadera e integralmente la labor profesional de los traductores e intérpretes, salvo los Decretos 382 y 2257 de 1951, que reglamentan la figura y ejercicio del intérprete oficial. Véanse Colombia, Presidencia de la República (1951a y 1951b).
2. Alta concentración del servicio en las dos ciudades principales (Bogotá y Medellín) y, en menor medida, en Cali, Barranquilla, Cartagena, Bucaramanga y Pereira, dejando desatendida una parte importante del territorio y, por consiguiente, encareciendo el servicio, o dejando en manos de personal sin idoneidad la realización de los encargos, particularmente de interpretación.

En consonancia con esta situación encontramos autores, como Aguayo (2013, p. 70), quien sostiene que, en el contexto español, particularmente en el de la comunidad autónoma de Andalucía, las pequeñas y medianas empresas por lo general recurren a traductores independientes. Los contratan en caso de que cobren barato o en caso de que se trate de un servicio en lenguas poco frecuentes. De lo contrario, recurren a los trabajadores con conocimientos de idiomas para realizar funciones de traducción e interpretación, aun sin formación previa en este campo. Según esta autora, se trata de una situación muy habitual y algunas de estas empresas parecen no estar dispuestas a querer cambiar este modo de proceder.

3. Gran número de profesionales se desempeñan como independientes, lo cual se percibe como trabajo informal, pues supone: a) percibir ingresos variables mes a mes, b) asumir los costos laborales relacionados con la seguridad social, y c) no percibir ciertas prestaciones laborales (primas de servicio, vacaciones, etc.). Además de lo anterior, trabajar como freelancer supone contar con pocos clientes y tener que asumir las labores propias de mercadeo para ampliar la base de clientes.

4. Bajo porcentaje de agremiación, lo que permite que haya cierta vulnerabilidad y desprotección a la hora de establecer tarifas y condiciones dignas para el ejercicio de la profesión. Este riesgo favorece la existencia del intrusismo y la competencia desleal.

Por último, coincidimos con Ortiz (2017, p. 85) quien, de modo general, sostiene que el mercado de los servicios de mediación intercultural es muy 
heterogéneo, caracterizado principalmente por la atomización y la disgregación de la oferta y la demanda, la complejidad variable de la cadena de producción e intermediación, la dependencia de la marcha de la economía, la especial propensión al intrusismo o la preponderancia de la competencia por precio.

$\mathrm{Al}$ realizar este estudio, nuestro propósito era despertar el interés en el sector como comunidad, para que los puntos débiles identificados se conviertan en oportunidades de mejora. En estudios posteriores se pretende abordar las diferenciaciones, por ejemplo, del tipo profesionales experimentados-recién graduados, con el fin de analizar las variables propuestas aquí y determinar cuán diferentes podrían llegar a ser los resultados.

Por todo lo anterior, hacemos un llamado vehemente a la comunidad académica, a las agremiaciones de traductores e intérpretes, y a los entes gubernamentales competentes para que, desde sus áreas de influencia, se trabaje mancomunadamente en un plan de acción a mediano plazo que permita subsanar estas carencias y lleve a la profesión a una posición más digna, sólida y visible en la sociedad. Solo así los profesionales de la traducción e interpretación lograrán aportar mayores beneficios a la sociedad, a su gremio y al sector productivo del país.

\section{Agradecimientos}

Los autores del artículo desean agradecer a la profesora Juana Mahissa Reyes Muñoz, de la Universidad Nacional, y a la traductora Jeannette Insignares Melo, de la Asociación Colombiana de Traductores, Terminólogos e Intérpretes (ACTTI), quienes hacen parte del comité técnico 218 del Icontec y fueron determinantes en la recolección de la información y el primer borrador del artículo.

\section{Referencias}

Aguayo, N. (2013). El traductor-intérprete en el comercio exterior: ¿realidad o necesidad? Entreculturas. Revista de Traducción y Comunicación Intercultural, (5), 57-74.

Aguayo, N. (2017). La interdisciplinariedad como elemento clave en la conceptualización de los estudios de traducción en el campo de conocimiento. Skopos. Revista internacional de Traducción, 8, 3-26. https:// www.uco.es/ucopress/ojs/index.php/skopos/article/ view/10519

Clavijo, S. (2018). Breaking language and cultural barriers: A key to improve stakeholder relationships. En R. Pérez, C. Salcedo y D. Ocampo (Eds.), Handbook of research on intrapreneurship and organizational sustainability in SMEs (pp.73-94). IGI Global. https:// doi.org/10.4018/978-1-5225-3543-0.ch004.

Clavijo, S. et al. (2008). Entorno de la traducción en Colombia. En S. Clavijo, E. Mendoza, C. A. Franco, M. I. Duque y E. Rodríguez (Eds.), Babel en las empresas colombianas: una mirada actual de la traducción (pp. 11-36). Ediciones Ean.

Clavijo, S., y Duro, M. (2020). Competitiveness in the language service industry in Colombia and Spain: Perspectives to increase productivity. En Handbook of research on increasing the competitiveness of SMEs (pp. 355-375). IGI global. https://doi.org/10.4018/978-1-5225-9425-3. ch016

Colombia, Congreso de la República (2012). Ley 1581, por la cual se dictan disposiciones generales para la protección de datos personales. http://www.secretariasenado.gov. co/senado/basedoc/ley_1581_2012.html

Colombia, Ministerio de Ciencia, Tecnología e Innovación (2020). Grupos de investigación en traducción. https://bit.ly/2VYFZO7

Colombia, Presidencia de la República (1951a). Decreto 382 , por el cual se crea el cargo de intérpretes oficiales. http://www.suin-juriscol.gov.co/viewDocument.asp? $\mathrm{id}=1086126$

Colombia, Presidencia de la República (1951b). Decreto 2257, por el cual se reglamenta el Decreto ley 382 del 19 de febrero de 1951, sobre intérpretes oficiales. https://www.mineducacion.gov.co/1759/w3-article-103361.html?_noredirect $=1$

Delisle, J., y Woodsworth, J. (Eds.) (2005). Los traductores en la historia. Editorial Universidad de Antioquia.

Departamento Administrativo Nacional de Estadística. (2012). Clasificación industrial internacional uniforme de todas las actividades económicas. https://bit.ly/2S05zR3

European Language Industry Association (2019). 2019 Language Industry Survey-Expectations and Concerns of the European Language Industry. https://bit.ly/2S0Un6r.

Fundación Italia Morayta. (2017). Estudio de encuesta sobre la traducción y la interpretación en México. Fundación Italia Morayta. https://bit.ly/3gJ2bE1 
Gouadec, D. (2010). Translation as a profession. John Benjamins. https://doi.org/10.1075/btl.73.

Hortal, A. (2010). Ética general de las profesiones (3. $\left.{ }^{\mathrm{a}} \mathrm{ed}.\right)$. Desclée de Brouwer.

ICONTEC. (2013). Norma técnica colombiana NTC-ISO 704 Trabajo terminológico. Principios y métodos. ICONTEC.

ICONTEC. (2017a). Norma técnica colombiana NTC-ISO 17100. Servicios de traducción. Requisitos para la prestación del servicio. ICONTEC.

ICONTEC. (2017b). Guía técnica colombiana GTC 283. Proyectos de traducción: Guia general. ICONTEC.

ICONTEC. (2018). Norma técnica colombiana NTC-ISO 18841: Servicios de interpretación, requisitos y recomendaciones generales. ICONTEC.

Lozano, C. (2011). Estudio sobre el mercado de la traducción en España [Tesis de grado, Universidad de Salamanca]. https://bit.ly/2RZDv01

Ministerio de Cultura de España y TT ACE Traductores (2010). Libro blanco de la traducción editorial en España. Ministerio de Cultura de España, TT ACE traductores. https://bit.ly/2zokFcy
Morón, M. (2010). Perfiles profesionales en traducción e interpretación: análisis DAFO en el marco de la sociedad multilingüe y multicultural. La Linterna del Traductor, 4, 91-109. https://bit.ly/2S0EahJ

Ortiz, J. (2017). Los estudios de traducción y el mundo hispánico: conceptos y ubicación. Matices en Lenguas Extranjeras, (10), 72-96. https://doi.org/10.15446/ male.n10.68180

Quiroz, G., Gómez, N. y Zuluaga, F. (2013). Panorama general del traductor e intérprete oficial en Colombia. Núcleo, 25(30), 165-203.

Quiroz, G., Zuluaga, F., y Gómez, N. (2015). Estatus actual del traductor en Colombia. Editorial Universidad de Antioquia.

Valdez, L. (2018). El traductor en Baja California. En L. R. Fernández (Coord.), La profesión del traductor en México (pp. 65-106). Universidad Intercontinental.

Zuluaga, J., y Quiroz, G. (2018). Análisis del desempeño de candidatos a traductor e intérprete oficial en Colombia. Cadernos de Tradução, 38(2), 263-293. https:// doi.org/10.5007/2175-7968.2018v38n2p263

Cómo citar este artículo: Giraldo-Ortiz, J. J., Clavijo-Olmos, B., Malavert-Chávez, C., SalazarGiraldo, B., Sarmiento-Jaramillo, C. (2020). Hacia una caracterización de los traductores e intérpretes en Colombia. Íkala, Revista de Lenguaje y Cultura, 25(3), 695-712. https:/doi.org/10.17533/udea. ikala.v25n03a01 